\title{
Analysis of Parameter Sensitivity Using Robust Design Techniques for a Flatfish Type Autonomous Underwater Vehicle
}

\author{
M. Santhakumar, ${ }^{1}$ T. Asokan, ${ }^{1}$ and T. R. Sreeram ${ }^{2}$ \\ ${ }^{1}$ Department of Engineering Design, Indian Institute of Technology Madras, Chennai 600036, India \\ ${ }^{2}$ A.P. Moller-Maersk Group, MAERSK LINE, Prince Infocity, 11th Floor 286/1, Old Mahabalipuram Road, \\ Kottivakkam-Kandanchavadi, Chennai 600096, India
}

Correspondence should be addressed to T. Asokan, asok@iitm.ac.in

Received 6 July 2009; Accepted 27 October 2009

Recommended by Suk joo Bae

Hydrodynamic parameters play a major role in the dynamics and control of Autonomous Underwater Vehicles (AUVs). The performance of an AUV is dependent on the parameter variations and a proper understanding of these parametric influences is essential for the design, modeling, and control of high-performance AUVs. In this paper, the sensitivity of hydrodynamic parameters on the control of a flatfish type AUV is analyzed using robust design techniques such as Taguchi's design method and statistical analysis tools such as Pareto-ANOVA. Since the pitch angle of an AUV is one of the crucial variables in the control applications, the sensitivity analysis of pitch angle variation is studied here. Eight prominent hydrodynamic coefficients are considered in the analysis. The results show that there are two critical hydrodynamic parameters, that is, hydrodynamic force and hydrodynamic pitching moment in the heave direction that influence the performance of a flatfish type AUV. A near-optimal combination of the parameters was identified and the simulation results have shown the effectiveness of the method in reducing the pitch error. These findings are significant for the design modifications as well as controller design of AUVs.

Copyright (C) 2009 M. Santhakumar et al. This is an open access article distributed under the Creative Commons Attribution License, which permits unrestricted use, distribution, and reproduction in any medium, provided the original work is properly cited.

\section{Introduction}

Autonomous Underwater Vehicles provide new alternatives for undersea exploration, relieving human divers from the risks and fatigue of working under constrained underwater environments. These are intelligent robots deployed to carry out predefined underwater tasks without human intervention. Since they are autonomous in nature, dynamics, control, and navigation of these robots are of major concern to the ocean engineering researchers. Developing a highly stable and maneuverable AUV with good payload capability is of major focus in the underwater robotics research today. The dynamic performance and autonomous control of an AUV depend on many factors such as robot shape, weight, buoyancy, propulsion, sensor systems, and control systems. The geometrical shape of the robot determines the hydrodynamic and added mass coefficients and has a very large influence on the robot dynamics $[1,2]$.
There are two major types of AUVs presently in use. The most common type is the torpedo-shaped AUV with a cylindrical hull [3], which is widely used in the design of AUVs. The flatfish type AUV has a geometric shape as shown in Figure 1, which is highly maneuverable and at the same time can carry higher payloads. This exceptionally stable shape produces low drag to the body and is wide enough to accept a variety of sensors [4].

The essential control requirements for any AUV are maneuvering in dive plane, depth control, and station keeping. While all these are critical requirements for an AUV, this paper focuses on the maneuvering in dive plane, especially the pitch angle variation during the forward motion of AUV. The design parameters that influence control requirements are the vehicle buoyancy, vertical distance between centre of gravity (CG) to centre of buoyancy $(\mathrm{CB})$, and the hydrodynamic parameters. Due to safety reasons, most of the AUVs are designed to have some minimum positive buoyancy. Similarly, the vertical distance between 


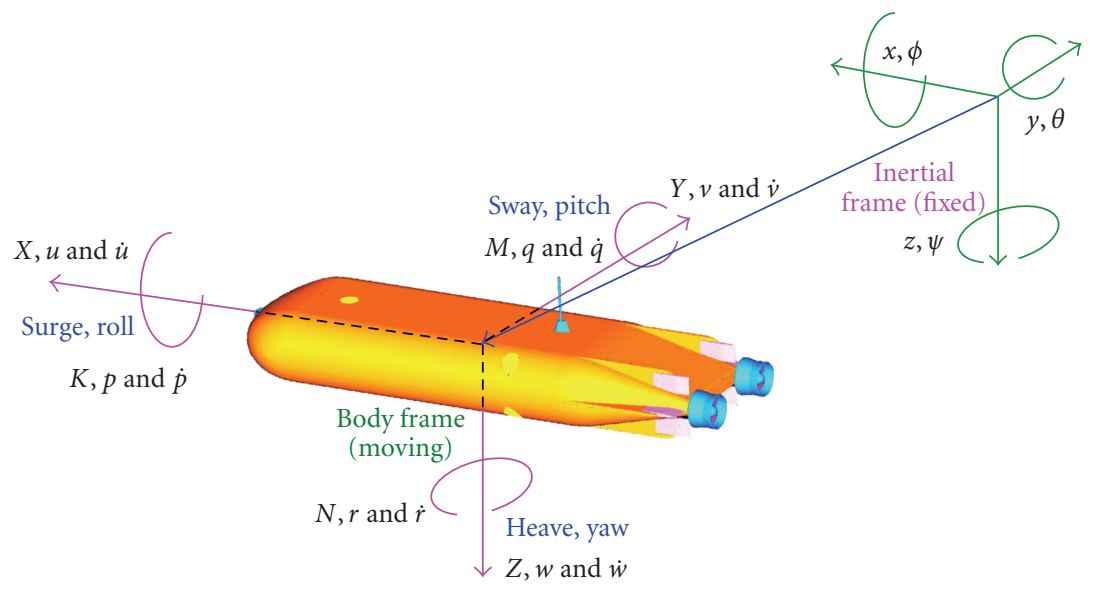

Figure 1: Body-fixed frame and earth-fixed reference frame for AUV.

CG and CB is always kept constant in order to ensure stability of the vehicle. Therefore, for a given configuration of AUV, the hydrodynamic parameter variation is the most critical and hence the sensitivity of these parameters in the pitch motion of an AUV is analyzed using Taguchi's design principles in this paper. The paper is organized in the following manner. A short introduction to the modeling and simulation of AUV with open-loop and closed-loop controls is presented in Section 2. Parametric model of an AUV is presented in Section 3, followed by a short summary of robust design formulation and an introduction to Taguchi techniques (Section 4 ). The sensitivity analysis and numerical simulations are presented in Section 5 for a flatfish type AUV. Finally the results are summarized.

\section{Modeling and Simulation}

Underwater robots experience a range of forces while moving in the fluid medium and they are generally referred to as hydrodynamic forces. A detailed discussion on hydrodynamic forces on underwater robots can be found in [5]. Added mass, drag, and lift are the most common reaction forces acting on the AUV and these are related to linear and angular accelerations and velocities. The hydrodynamic parameters relate these forces to the corresponding robot parameters that are highly nonlinear. Many of these parameters are dependent on the shape of AUV. In the case of flatfish type AUV, these hydrodynamic parameters are not easily derived, owing to the complex nature of the geometry. Since the robot performance depends on these parameters, it is necessary to identify the critical parameters and control them to improve vehicle performance. To investigate the dynamic behavior of the AUV, a mathematical model of the robot has been developed. The dynamic motion of AUV can be described in a common way using six degrees of freedom (DOF) nonlinear equations (refer to Figure 1). The equations of motion of the AUV can be written as in $[5,6]$ and these equations show the various parameters which are involved in the dynamic motion of the robot and are used in the numerical simulation. The simulation method and its details are given in the proceeding section.
Surge Motion:

$$
\begin{aligned}
(m & \left.-X_{\dot{u}}\right) \dot{u}-\left(X_{u}+X_{u|u|}|u|\right) u-\left(X_{v}+X_{v|v|}|v|\right) v \\
& -\left(X_{w}+X_{w|w|}|w|\right) w-\left(m x_{G}+Z_{\dot{q}}\right) q^{2}-\left(m x_{G}-Y_{\dot{r}}\right) r^{2} \\
& +\left(Y_{\dot{v}}-m\right) v r+\left(m-Z_{\dot{w}}\right) w q+(W-B) \sin \theta \\
= & \left(X_{\delta s}+X_{\delta s|\delta s|}|\delta s|\right) \delta s+\left(X_{\delta r}+X_{\delta r|\delta r|}|\delta r|\right) \delta r+\tau_{\text {prop }} .
\end{aligned}
$$

Sway Motion:

$$
\begin{aligned}
\left(m-Y_{\dot{v}}\right) \dot{v}-\left(Y_{v}+Y_{v|v|}|v|\right) v \\
\quad-Y_{r} r+\left(m x_{G}-Y_{\dot{r}}\right) \dot{r}+m u r+\left(m x_{G}+Z_{\dot{q}}\right) p q \\
\quad+\left(m-Z_{\dot{w}}\right) w p-(W-B) \cos \theta \sin \phi \\
=\left(Y_{\delta r}+Y_{\delta r \mid}|\delta r| \delta r \mid\right) \delta r .
\end{aligned}
$$

Heave Motion:

$$
\begin{aligned}
& \left(m-Z_{\dot{w}}\right) \dot{w}-\left(Z_{w}+Z_{w|w|}|w|\right) w \\
& \quad-Z_{q} q+\left(m x_{G}-Z_{\dot{q}}\right) \dot{q}-m u q+\left(m x_{G}-Y_{\dot{r}}\right) r p \\
& \quad+\left(m-Y_{\dot{v}}\right) v p-(W-B) \cos \theta \cos \phi \\
& =\left(Z_{\delta s}+Z_{\delta s|\delta s|}|\delta s|\right) \delta s .
\end{aligned}
$$

Roll Motion:

$$
\begin{aligned}
& \left(I_{x}-K_{\dot{p}}\right) \dot{p}+\left(I_{z}-I_{y}\right) q r \\
& \quad+\left(Y_{\dot{v}}-Z_{\dot{w}}\right) v w-Z_{\dot{q}} v q+\left(M_{\dot{q}}-N_{\dot{r}}\right) r q+Y_{\dot{r}} r w \\
& \quad-K_{p} p-z_{B} B \cos \theta \sin \phi=0 .
\end{aligned}
$$


Pitch Motion:

$$
\begin{aligned}
& \left(I_{y}-M_{\dot{q}}\right) \dot{q}-m x_{G} \dot{w}+m x_{G} u q \\
& \quad+\left(I_{x}-I_{z}+N_{\dot{r}}\right) r p-m x_{G} v p-\left(M_{w}+M_{w|w|}|w|\right) w \\
& \quad-M_{q} q-z_{B} B \sin \theta+\left(x_{G} W-x_{B} B\right) \cos \theta \cos \phi \\
& =\left(M_{\delta s}+M_{\delta s|\delta s|}|\delta s|\right) \delta s .
\end{aligned}
$$

Yaw Motion:

$$
\begin{aligned}
& \left(I_{z}-N_{\dot{r}}\right) \dot{r}-\left(N_{v}+N_{v|v|}|v|\right) v \\
& \quad-N_{r} r+m x_{G} u r+\left(I_{y}-I_{x}-M_{\dot{q}}\right) p q-m x_{G} w p \\
& \quad-\left(x_{G} W-x_{B} B\right) \cos \theta \sin \phi=\left(N_{\delta r}+N_{\delta r|\delta r|}|\delta r|\right) \delta r,
\end{aligned}
$$

where $\left[\begin{array}{llllll}x & y & z & \phi & \theta & \psi\end{array}\right]$ are positions and orientations with respect to inertial (fixed) frame, $x$ is surge position, $y$ is sway position, $z$ is heave position, $\phi$ is roll angle, $\theta$ is pitch angle, and $\psi$ is yaw angle, $\left[\begin{array}{llllll}u & v & w & p & q & r\end{array}\right]$ are linear and angular velocities with respect to body (moving) frame, $u$ is surge velocity, $v$ is sway velocity, $w$ is heave velocity, $p$ is roll rate, $q$ is pitch rate, $r$ is yaw rate. $\dot{u}, \dot{v}$ and $\dot{w}$ are linear accelerations in surge, sway, and heave directions, respectively. $\dot{p}, \dot{q}$, and $\dot{r}$ are angular accelerations in roll, pitch, and yaw directions, respectively. [ $\left[\begin{array}{llllll}X & Y & Z & K & M & N\end{array}\right]$ are forces and moments (which include hydrodynamic damping and added mass) acting on AUV (such as $X_{u|u|}, X_{u}, X_{\dot{u}}$ ), $X$ is resultant force in surge direction, $Y$ is resultant force in sway direction, $Z$ is resultant force in heave direction, $K$ is resultant moment in roll axis, $M$ is resultant moment in pitch axis, $N$ is resultant moment in yaw axis, $m$ is mass of the AUV, $W$ is weight of the AUV, $B$ is buoyancy of the AUV, $I$ is inertia terms of the AUV, $\delta s$ is stern control plane deflection, $\delta r$ is rudder plane deflection, $C_{G}=\left[x_{G}, y_{G}, z_{G}\right]=$ Centre of Gravity, and $C_{B}=\left[x_{B}, y_{B}, z_{B}\right]=$ Centre of Buoyancy.

2.1. Initial Study. In order to identify the sensitivity of these parameters, an experimental AUV that is being developed at the Indian Institute of Technology, Madras, India, is considered a test case. The dynamic model of the robot is developed using the Newton-Euler formulation [5] and is simulated using the experimental robot (positive buoyant) parameters and the experimentally determined hydrodynamic parameters. Simulation experiments were carried out with the help of MATLAB-Simulink software (step size is $0.01 \mathrm{~s}$ ) and Runge-Kutta's fourth-order model (RK4 solver is used) for the above dynamic equations. Repeated sequences of random signals (white noises) are considered the noise effects, which are introduced in the input stage and their amplitudes are limited by \pm 2 units. The results are shown in Figure 2. It shows a large steady state pitch angle during the forward motion, which is not acceptable from the point of view of control and navigation. This large steady state pitch angle results in the heave and sway motions also (refer to Figure 2), which is, again, not desirable. One option at this stage is to go for closed loop control of the AUV, which will reduce the pitch error. However, the control forces needed to achieve this will be very high and eventually lead to poor overall performance. Hence, it is necessary to identify the causes for pitch variation and rectify the problem through redesign of the AUV. Sensitivity analysis is one of the methods to identify the critical hydrodynamic parameters that contribute to the pitching motion. Robust design methods, developed for general design optimization, can be effectively used here to analyze the sensitivity of the AUV to various hydrodynamic parameters.

\section{AUV Parametric Model}

A parametric model of the AUV for the pitch motion is developed to study the pitch angle variation and the parameter sensitivity.

3.1. AUV Model for Pitch. With reference to Figure 1, the pitch motion is with respect to the $Y$ axis, in the vertical plane. Assuming that $\left(x_{B}, y_{B}, z_{B}\right)$ and $\left(x_{G}, y_{G}, z_{G}\right)$ be the coordinates of centre of buoyancy and centre of gravity of the vehicle with respect to the body centre, the AUV model equations for pitch are derived as follows $[5,6]$ :

$$
\begin{aligned}
& \left(\begin{array}{c}
\dot{w} \\
\dot{q} \\
\dot{z} \\
\dot{\theta}
\end{array}\right) \\
& =M^{-1}\left[\begin{array}{cccc}
Z_{w} & Z_{q}-\left(m z_{G} q+m u\right)-X_{\dot{u}} u & 0 & 0 \\
M_{w}+X_{\dot{u}} u & M_{q}+m\left(x_{G} u+z_{G} w\right) & 0 & 0 \\
\cos \theta & 0 & 0 & 0 \\
0 & 1 & 0 & 0
\end{array}\right]\left(\begin{array}{c}
w \\
q \\
z \\
\theta
\end{array}\right) \\
& +M^{-1}\left(\begin{array}{c}
(W-B) \cos \theta \\
\left(x_{B} B-x_{G} W\right) \cos \theta+\left(z_{B} B-z_{G} W\right) \sin \theta \\
-u \sin \theta \\
0
\end{array}\right) \\
& +M^{-1}\left(\begin{array}{c}
Z_{\delta} \\
M_{\delta} \\
0 \\
0
\end{array}\right)
\end{aligned}
$$

where inertia matrix is

$$
M=\left[\begin{array}{cccc}
m-Z_{\dot{w}} & -m x_{G}-Z_{\dot{q}} & 0 & 0 \\
-m x_{G} & I_{y y}-M_{\dot{q}} & 0 & 0 \\
0 & 0 & 1 & 0 \\
0 & 0 & 0 & 1
\end{array}\right] .
$$

The terms $X_{\dot{u}}, Z_{\dot{w}}, Z_{\dot{q}}, M_{\dot{q}}, Z_{w}, Z_{q}, M_{w}$, and $M_{q}$ in (7) are the hydrodynamic parameters (hydrodynamic damping and 


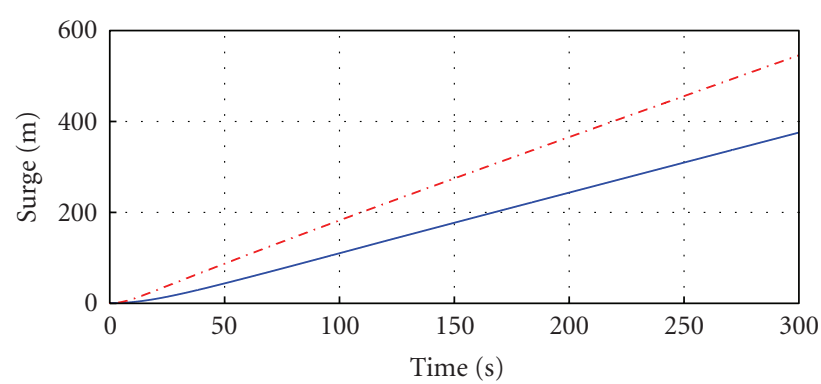

(a)

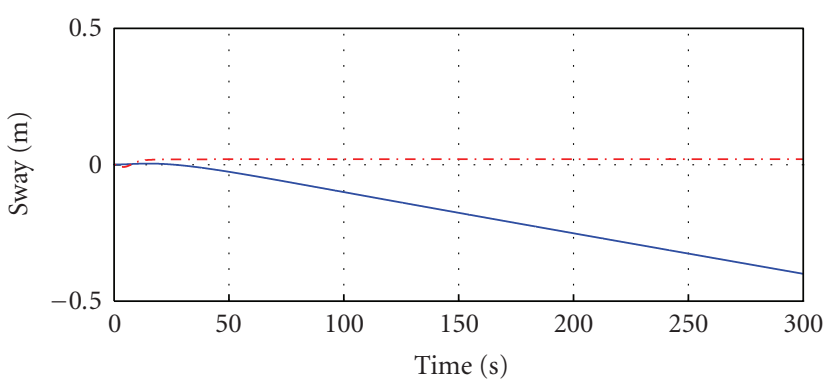

(c)

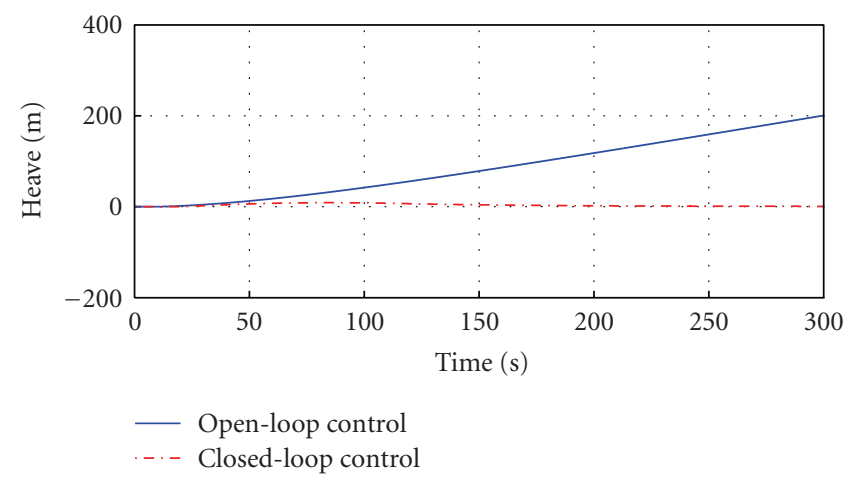

(e)

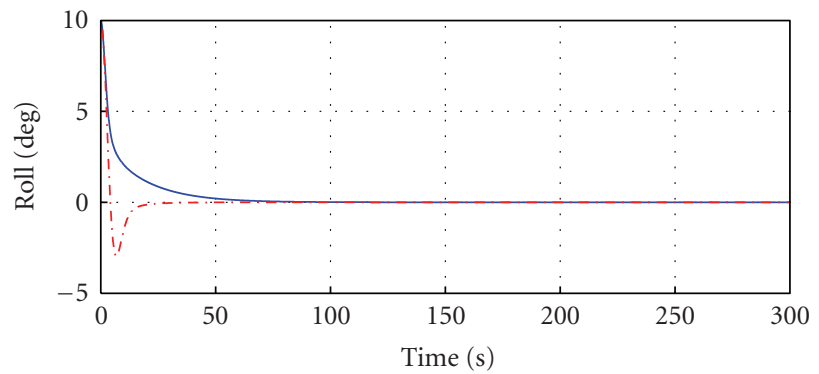

(b)

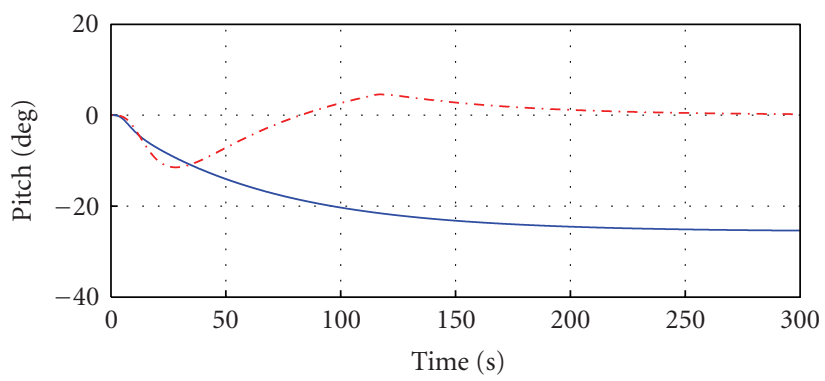

(d)

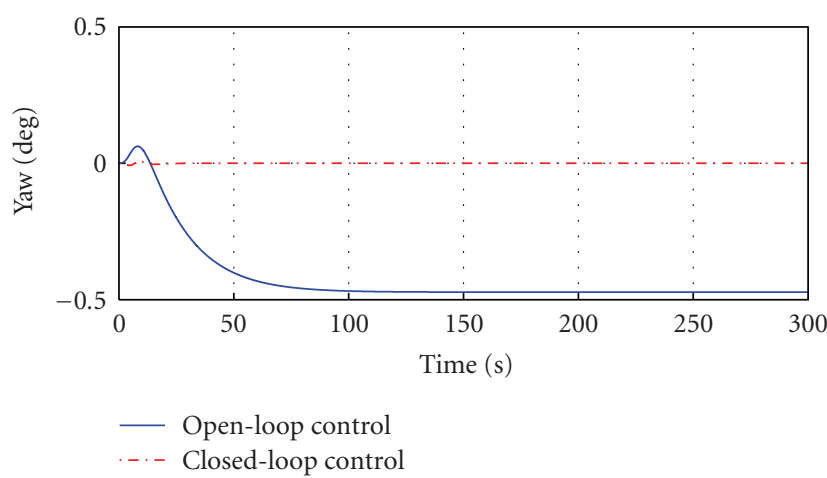

(f)

Figure 2: AUV positions and orientations for constant forward speed (at $U=1.98 \mathrm{~m} / \mathrm{s}$ ).

added mass forces and moments) affecting the pitch motion. Here, $X_{\dot{u}}, Z_{\dot{w}}$, and $Z_{\dot{q}}$ are the added mass forces due to accelerations $\dot{u}, \dot{w}$, and $\dot{q}$, respectively, and $M_{\dot{q}}$ is the hydrodynamic added mass moment due to acceleration $\dot{q}$ along the pitch axis. Similarly $Z_{w}$ and $Z_{q}$ are the hydrodynamic damping forces along the $Z$ axis due to velocities $w$ and $q$, and $M_{w}$ and $M_{q}$ are the hydrodynamic damping moments in the pitch axis due to velocities $w$ and $q$.

\section{Robust Design Formulation}

Engineering design is increasingly becoming model based, in that its complexity calls for a mathematical model involving multiple quantities, some of which are to be decided by the designer with the purpose of meeting performance specifications, for example, the thrust that an underwater propeller must deliver at a given rpm, under given environment conditions such as ambient temperature, pressure, and so forth. The aim of robust design is to develop products whose performance remains within specifications in the presence of large variations in environment conditions.

4.1. Sensitivity Analysis. It is a procedure to determine the sensitivity of the outcomes of an alternative to changes in its parameters (as opposed to changes in the environment). If a small change in a parameter results in relatively large change in the outcomes, the outcomes are said to be sensitive to that parameter. This may mean that the parameter has to be determined very accurately or the alternative is to redesign such that the sensitivity of the parameter is low $[6,7]$. Sensitivity Analysis can be used to determine

(1) the model resemblance with the system under study,

(2) the quality of model definition,

(3) factors that mostly contribute to the output variability, 
(4) the region in the space of input factors for which the model variation is maximum,

(5) interactions between factors.

Parameter sensitivity is usually performed as a series of tests in which the modeler sets different parameter values to see how a change in the parameter causes a change in the dynamic behavior of the system. By showing how the model behavior responds to change in parameter values, sensitivity analysis is a useful tool in model building as well as in model evaluation $[8,9]$.

For sensitivity analysis, Taguchi's robust design is more appropriate because it is a design and data analysis method and is more engineering oriented than science oriented. The distinct idea of Taguchi's robust design that differs from the conventional experimental design is that of designing for the simultaneous modeling of both mean and variability [10].

The concept of robust design has many aspects such as

(i) finding a set of conditions for design variables which are robust to noise,

(ii) achieving the smallest variation in a product's function relative to a desired target value,

(iii) minimizing the number of experiments using orthogonal arrays and testing for confirmation.

4.2. Robust Design. Robust Design is a method, also called the Taguchi Method, pioneered by Byrne and Taguchi [11] that greatly improves engineering productivity. Taguchi introduced the concept of parameter design as an effective means of improving the efficiency of a system which involves significant variability or "noise." A system can perform its intended function at many settings/values of design parameters. The method requires the consideration of the effect of noise factors (environmental variation during the system's usage, manufacturing variation of the system, and component deterioration) and the cost of failure in the field to ensure the performance requirements of the system. Indeed, it is the most powerful method available to reduce the variation, improve efficiency, and simultaneously reduce development interval [10]. Robust design technique can be applied to many aspects such as optimization, experimental design, sensitivity analysis, parameter estimation, model prediction, and so forth $[7,10]$.

4.3. Taguchi's Approach. In setting up a framework for robust design, the classification of the quantities is at play in the design task as given below.

(i) Design variables (DVs) are those quantities to be decided by the designer with the purpose of meeting performance specifications under given conditions.

(ii) Design-environment parameters (DEPs) are the quantities over which the designer has no control and define the conditions of the environment under which the designed object will operate.

(iii) Performance functions (PFs) are quantities used to represent the performance of the design in terms

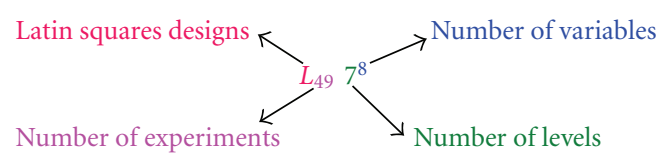

Figure 3: Representation of standard orthogonal array.

of design variables and design-environment parameters.

The responses at each setting of parameters are treated as a measure that would be indicative of not only the mean of some quality characteristic but also the variance of the same characteristic. The mean and the variance are combined into a single performance measure known as the signal-to-noise $(\mathrm{S} / \mathrm{N})$ ratio [10]. Taguchi classifies robust parameter design problems into different categories depending on the goal of the problem and for each category as follows.

Smaller the better: the target value of $y$, that is, quality variable, is zero. In this situation, $\mathrm{S} / \mathrm{N}$ ratio is defined as follows:

$$
\mathrm{S} / \mathrm{N} \text { Ratio }=-10 \log \left(\frac{1}{n} \sum_{i=1}^{n} y_{i}^{2}\right)
$$

Larger the better: the target value of $y$, that is, quality variable is infinite and $\mathrm{S} / \mathrm{N}$ ratio is defined as follows:

$$
\mathrm{S} / \mathrm{N} \text { Ratio }=-10 \log \left(\frac{1}{n} \sum_{i=1}^{n} \frac{1}{y_{i}^{2}}\right) .
$$

Nominal the best: the certain target value is given for $y$ value. In this situation, $\mathrm{S} / \mathrm{N}$ ratio is defined as follows:

$$
\mathrm{S} / \mathrm{N} \text { Ratio }=-10 \log \left(\sum_{i=1}^{n} \frac{y_{i}^{2}}{s^{2}}\right) .
$$

In this paper, smaller the better characteristic is used due to the requirement to keep the pitch angle variation to a minimum.

Taguchi's method uses an orthogonal array (OA) and analysis of mean to study the effects of parameters based on statistical analysis of experiments. An OA is a fractional factorial matrix which assures a balanced comparison of levels of any factor or interaction of factors. It is a matrix of numbers arranged in rows and columns where each row represents the level of the factors in each run, and each column represents a specific factor that can be changed from each run. The array is called orthogonal because all columns can be evaluated independently of one another. To compare performances of parameters, the statistical test known as the analysis of variance (ANOVA) is used. Further details and technical merits about robust parameter design can be found in the references of $[9,11-14]$. The standard array representation of OA is depicted in Figure 3. Standard OA is basically derived from linear graph of number of variables and levels; the ones most often used are $L_{4}\left(2^{3}\right), L_{8}\left(2^{7}\right), L_{9}$ $\left(3^{4}\right), L_{25}\left(5^{6}\right)$, and $L_{49}\left(7^{8}\right)[7]$. 


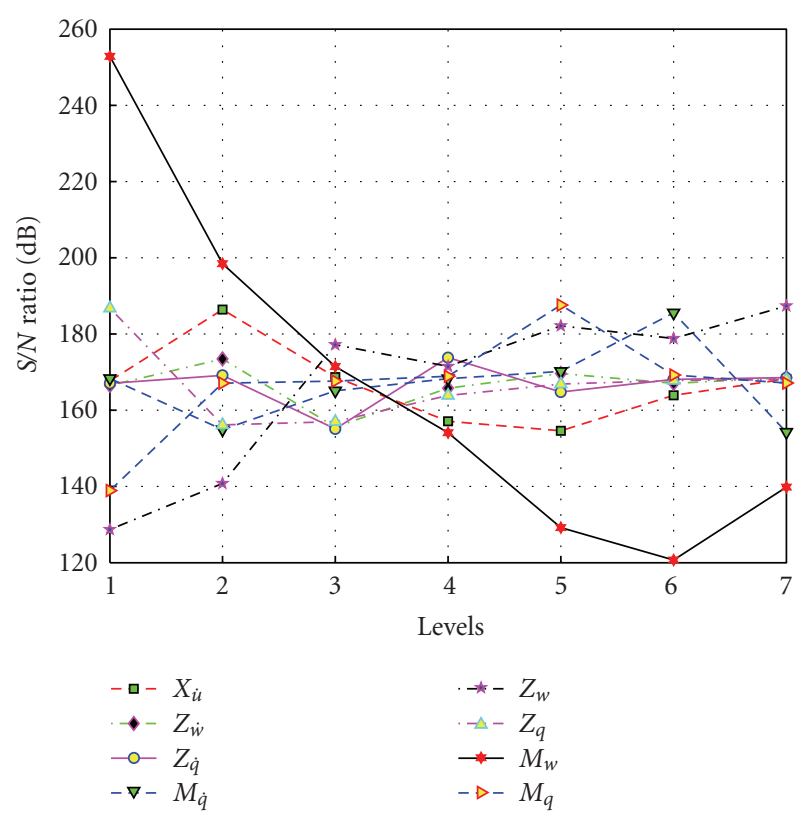

Figure 4: Response curves.

Once the suitable orthogonal array for experimentation is selected, then the experiments are conducted for the identified conditions and the results are analyzed using statistical methods, such as Pareto-ANOVA, ANOVA, or response curves to identify the optimal parameters for performance enhancement $[7,11,15,16]$.

\section{Numerical Simulation}

As explained in Sections 2 and 3, the objective of the work is to identify the critical hydrodynamic parameters influencing the pitch angle (performance function) variation of the AUV. As explained in Section 2.1, there are eight hydrodynamic parameters (design variables) that influence the pitching moment of the robot (AUV). As these parameters are functions of forward speed of the vehicle, this factor is used as the noise factor (design environment parameter) in the analysis. This noise factor (forward velocity of the vehicle) is changed by providing different thrust values in the simulation such as $25 \%, 50 \%$, and $75 \%$ of total thrust available from the actuators. The details of the analysis and the results are presented in the following sections.

5.1. Experimental Layout. The first step in the analysis is to choose the orthogonal array for trials. For selecting the orthogonal array, the main criterion is the required number of trials [7].

Mathematically, this is given as

$$
N=A(L-1),
$$

where $N=$ Number of experiments, $A=$ Number of variables, and $L=$ Number of levels.

It was decided to conduct trials using the eight variables at seven levels (more numbers of levels means, more number of experiments, and better accuracy of results), that is, $10 \%$, $25 \%, 40 \%, 55 \%, 70 \%, 85 \%$, and $100 \%$ of the actual values of the variables. So, the number of trials required was found to be 49 . The smallest standard orthogonal array that matches with this requirement is $L_{49}\left(7^{8}\right)$, which will give a test matrix of 49 different configurations with various combinations of parameters and its physical values (eight parameters at seven levels). Three levels of noise variable (vehicle speed) are considered in the analysis and the test matrix used is shown in Table 1.

\section{Results and Discussions}

The simulation trials were conducted for the states mentioned in the OA. For each experiment, the pitch angle was recorded (refer to Table 1 ) and the SNR (S/N ratio) (using smaller the better characteristics) was calculated. For example, the SNR for experiment 1 was calculated as

$$
\begin{aligned}
\mathrm{SNR}= & -10 \log \left(\frac{1}{n} \sum_{i=1}^{n} y_{i}^{2}\right) \\
= & -10 \log \left(\frac{1}{3}\left[(-8.82)^{2}+(-10.03)^{2}+(-10.93)^{2}\right]\right) \\
& +50=30.03 \mathrm{~dB} .
\end{aligned}
$$

( $50 \mathrm{~dB}$ is added to avoid negative values for $\mathrm{S} / \mathrm{N}$ ratio, to make comparisons easy).

The plot of SNR for various parameters is shown in Figure 4, which indicates a larger variation for $M_{w}$ and $Z_{w}$ and higher sensitivity of these parameters.

In order to identify the optimal parameter combination and the contribution ratio of each parameter, statistical analysis of the results was carried out using Pareto-ANOVA analysis.

For Pareto-ANOVA analysis, the sum of $\mathrm{S} / \mathrm{N}$ ratios, sum of squares of differences, and contribution ratio were calculated as follows:

$$
\begin{gathered}
S_{i j}=\sum(\mathrm{SNR})_{i j}, \\
S S_{i}=\sum_{j=2}^{7}\left(S_{i 1}-S_{i j}\right)^{2}, \\
C R_{i}=\frac{S S_{i}}{\sum S S_{i}} \times 100,
\end{gathered}
$$

where $S_{i j}=$ Sum of SNR of $i$ th parameter at $j$ th level, $(\mathrm{SNR})_{i j}$ $=\mathrm{S} / \mathrm{N}$ ratio of $i$ th parameter at $j$ th level, $S S_{i}=$ Sum of squares of differences for $i$ th parameter, and $C R_{i}=$ Percentage of contribution ratio.

For example, the calculation of above values for $M_{w}$ is shown as follows:

Sum of the S/N ratio for Level 1 of $M_{\mathrm{w}}$

$$
\begin{aligned}
= & 30.03+39.61+40.72+32.62+34.86 \\
& +36.72+38.27=252.83 \mathrm{~dB} .
\end{aligned}
$$


TABle 1: Design of Experiments for Pitch Response $\left(L_{49}\left(7^{8}\right) \mathrm{OA}\right)$.

\begin{tabular}{|c|c|c|c|c|c|c|c|c|c|c|c|c|}
\hline \multirow{2}{*}{ Exp. no. } & \multirow{2}{*}{$\begin{array}{c}X_{\dot{u}} \\
(\%) \\
\end{array}$} & \multirow{2}{*}{$\begin{array}{c}Z_{\dot{w}} \\
(\%)\end{array}$} & \multirow{2}{*}{$\begin{array}{c}Z_{\dot{q}} \\
(\%) \\
\end{array}$} & \multirow{2}{*}{$\begin{array}{c}M_{\dot{q}} \\
(\%) \\
\end{array}$} & \multirow{2}{*}{$\begin{array}{c}Z_{w} \\
(\%)\end{array}$} & \multirow{2}{*}{$\begin{array}{c}Z_{q} \\
(\%)\end{array}$} & \multirow{2}{*}{$\begin{array}{l}M_{w} \\
(\%)\end{array}$} & \multirow{2}{*}{$\begin{array}{l}M_{q} \\
(\%)\end{array}$} & \multicolumn{3}{|c|}{ Pitch angle (deg) for $U=$} & \multirow{2}{*}{$\mathrm{S} / \mathrm{N}$ ratio $(\mathrm{dB})$} \\
\hline & & & & & & & & & $1.13(\mathrm{~m} / \mathrm{s})$ & $1.59(\mathrm{~m} / \mathrm{s})$ & $1.98(\mathrm{~m} / \mathrm{s})$ & \\
\hline 1 & 10 & 10 & 10 & 10 & 10 & 10 & 10 & 10 & -8.82 & -10.03 & -10.93 & 30.03 \\
\hline 2 & 10 & 25 & 25 & 40 & 55 & 70 & 85 & 100 & -33.43 & -34.46 & -34.97 & 19.30 \\
\hline 3 & 10 & 40 & 40 & 55 & 70 & 85 & 100 & 25 & -32.63 & -33.33 & -33.67 & 19.57 \\
\hline 4 & 10 & 55 & 55 & 70 & 85 & 100 & 25 & 40 & -7.82 & -7.98 & -8.06 & 31.99 \\
\hline 5 & 10 & 70 & 70 & 85 & 100 & 25 & 40 & 55 & -10.73 & -10.88 & -10.97 & 29.28 \\
\hline 6 & 10 & 85 & 85 & 100 & 25 & 40 & 55 & 70 & -35.01 & -37.61 & -39.08 & 18.57 \\
\hline 7 & 10 & 100 & 100 & 25 & 40 & 55 & 70 & 85 & -34.31 & -35.91 & -36.73 & 18.96 \\
\hline 8 & 25 & 10 & 25 & 25 & 25 & 25 & 25 & 25 & -17.12 & -18.56 & -19.48 & 24.70 \\
\hline 9 & 25 & 25 & 55 & 85 & 40 & 10 & 100 & 70 & -5.42 & -5.76 & -5.96 & 34.86 \\
\hline 10 & 25 & 40 & 85 & 40 & 10 & 100 & 70 & 55 & -53.19 & -58.33 & -60.97 & 14.79 \\
\hline 11 & 25 & 55 & 40 & 10 & 100 & 70 & 55 & 85 & -14.53 & -14.73 & -14.83 & 26.66 \\
\hline 12 & 25 & 70 & 10 & 100 & 70 & 55 & 85 & 40 & -28.52 & -29.16 & -29.49 & 20.73 \\
\hline 13 & 25 & 85 & 100 & 70 & 55 & 85 & 40 & 10 & -17.15 & -17.76 & -18.11 & 25.05 \\
\hline 14 & 25 & 100 & 70 & 55 & 85 & 40 & 10 & 100 & -3.25 & -3.32 & -3.35 & 39.61 \\
\hline 15 & 40 & 10 & 40 & 40 & 40 & 40 & 40 & 40 & -21.04 & -22.14 & -22.78 & 23.15 \\
\hline 16 & 40 & 25 & 85 & 70 & 100 & 55 & 10 & 25 & -2.86 & -2.92 & -2.95 & 40.72 \\
\hline 17 & 40 & 40 & 70 & 100 & 55 & 10 & 25 & 85 & -10.93 & -11.33 & -11.57 & 28.95 \\
\hline 18 & 40 & 55 & 100 & 55 & 10 & 25 & 85 & 70 & -61.21 & -63.12 & -65.36 & 13.98 \\
\hline 19 & 40 & 70 & 55 & 10 & 25 & 85 & 70 & 100 & -42.29 & -45.13 & -46.61 & 16.99 \\
\hline 20 & 40 & 85 & 10 & 25 & 85 & 70 & 100 & 55 & -28.45 & -28.92 & -29.15 & 20.80 \\
\hline 21 & 40 & 100 & 25 & 85 & 70 & 100 & 55 & 10 & -19.36 & -19.83 & -20.09 & 24.08 \\
\hline 22 & 55 & 10 & 55 & 55 & 55 & 55 & 55 & 55 & -23.01 & -23.79 & -24.23 & 22.51 \\
\hline 23 & 55 & 25 & 40 & 100 & 85 & 25 & 70 & 10 & -89.98 & -89.98 & -89.98 & 10.92 \\
\hline 24 & 55 & 40 & 100 & 85 & 25 & 70 & 10 & 40 & -6.82 & -7.45 & -7.87 & 32.62 \\
\hline 25 & 55 & 55 & 85 & 25 & 70 & 10 & 40 & 100 & -14.35 & -14.71 & -14.86 & 26.69 \\
\hline 26 & 55 & 70 & 25 & 70 & 10 & 40 & 100 & 85 & -65.01 & -67.02 & -69.24 & 13.46 \\
\hline 27 & 55 & 85 & 70 & 10 & 40 & 100 & 85 & 25 & -39.9 & -41.59 & -42.43 & 17.68 \\
\hline 28 & 55 & 100 & 10 & 40 & 100 & 85 & 25 & 70 & -6.83 & -6.93 & -6.99 & 33.20 \\
\hline 29 & 70 & 10 & 70 & 70 & 70 & 70 & 70 & 70 & -24.09 & -24.66 & -24.96 & 22.19 \\
\hline 30 & 70 & 25 & 10 & 55 & 25 & 100 & 40 & 85 & -26.58 & -28.72 & -30.01 & 20.91 \\
\hline 31 & 70 & 40 & 55 & 25 & 100 & 40 & 85 & 10 & -89.98 & -89.98 & -89.98 & 10.92 \\
\hline 32 & 70 & 55 & 25 & 100 & 40 & 85 & 10 & 55 & -5.42 & -5.76 & -5.96 & 34.86 \\
\hline 33 & 70 & 70 & 100 & 40 & 85 & 10 & 55 & 25 & -16.63 & -16.93 & -17.09 & 25.45 \\
\hline 34 & 70 & 85 & 40 & 85 & 10 & 55 & 25 & 100 & -22.44 & -25.36 & -27.41 & 21.99 \\
\hline 35 & 70 & 100 & 85 & 10 & 55 & 25 & 100 & 40 & -37.96 & -38.11 & -39.23 & 18.30 \\
\hline 36 & 85 & 10 & 85 & 85 & 85 & 85 & 85 & 85 & -24.73 & -25.15 & -25.37 & 22.01 \\
\hline 37 & 85 & 25 & 100 & 10 & 70 & 40 & 25 & 55 & -9.13 & -9.38 & -9.51 & 30.59 \\
\hline 38 & 85 & 40 & 10 & 70 & 40 & 25 & 55 & 100 & -28.02 & -29.41 & -30.18 & 20.69 \\
\hline 39 & 85 & 55 & 70 & 40 & 25 & 55 & 100 & 10 & -89.98 & -89.98 & -89.98 & 10.92 \\
\hline 40 & 85 & 70 & 40 & 25 & 55 & 100 & 10 & 70 & -4.45 & -4.64 & -4.75 & 36.72 \\
\hline 41 & 85 & 85 & 25 & 55 & 100 & 10 & 70 & 40 & -18.22 & -18.46 & -18.59 & 24.69 \\
\hline 42 & 85 & 100 & 55 & 100 & 10 & 70 & 40 & 25 & -34.53 & -38.84 & -41.59 & 18.31 \\
\hline 43 & 100 & 10 & 100 & 100 & 100 & 100 & 100 & 100 & -25.13 & -25.46 & -25.62 & 21.90 \\
\hline 44 & 100 & 25 & 70 & 25 & 10 & 85 & 55 & 40 & -44.8 & -49.85 & -52.76 & 16.15 \\
\hline 45 & 100 & 40 & 25 & 10 & 85 & 55 & 40 & 70 & -12.3 & -12.53 & -12.65 & 28.07 \\
\hline 46 & 100 & 55 & 10 & 85 & 55 & 40 & 70 & 25 & -28.45 & -29.38 & -29.86 & 20.68 \\
\hline 47 & 100 & 70 & 85 & 55 & 40 & 70 & 25 & 10 & -13.46 & -14.2 & -14.64 & 27.01 \\
\hline 48 & 100 & 85 & 55 & 40 & 70 & 25 & 10 & 85 & -3.76 & -3.87 & -3.94 & 38.27 \\
\hline 49 & 100 & 100 & 40 & 70 & 25 & 10 & 85 & 55 & -48.52 & -49.12 & -51.12 & 16.09 \\
\hline
\end{tabular}




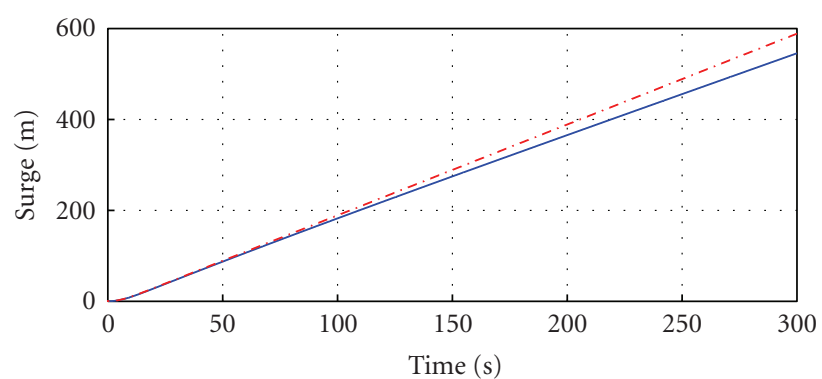

(a)

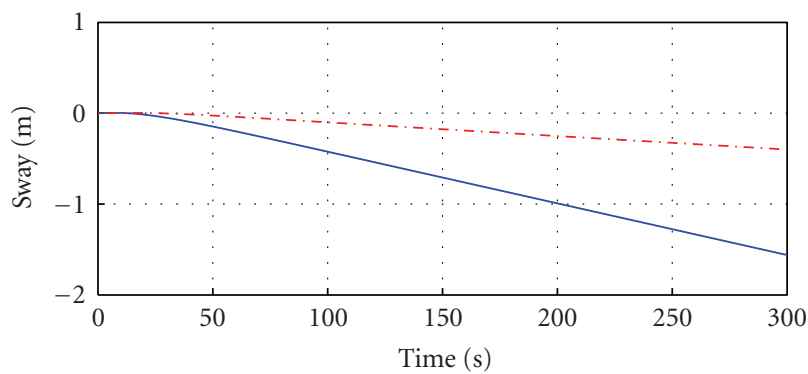

(c)

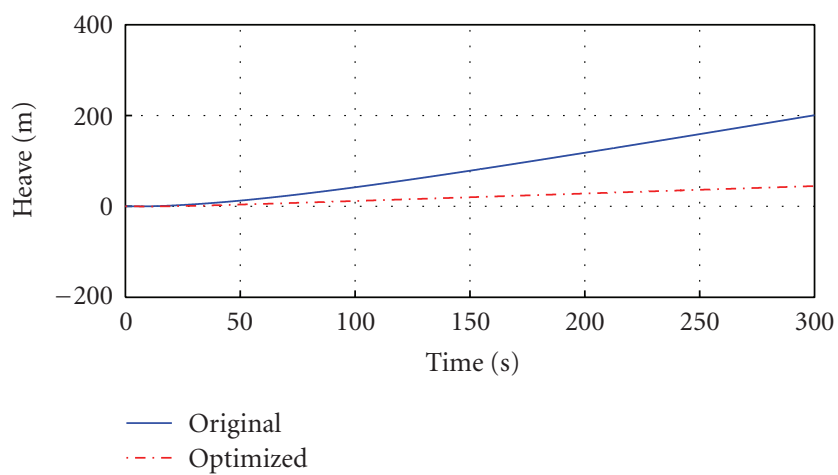

(e)

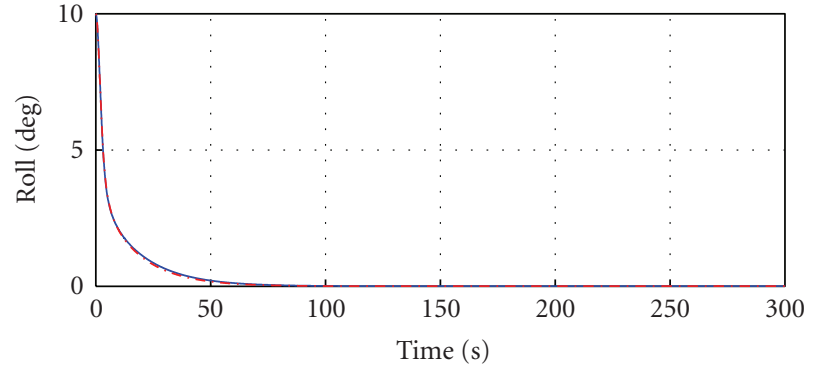

(b)

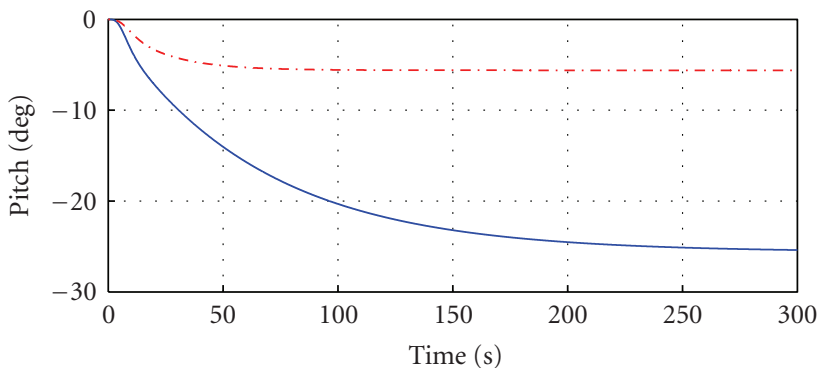

(d)

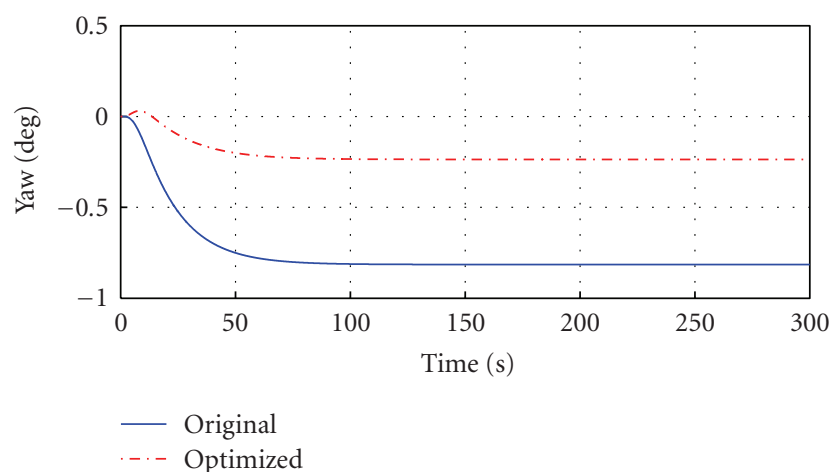

(f)

FIgURE 5: Comparative results of AUV positions and orientations for open loop control.

Sum of squares of Differences of $M_{\mathrm{W}}$

$$
\begin{aligned}
= & (252.83-198.43)^{2}+(252.83-171.46)^{2} \\
& +(252.83-154.11)^{2}+(252.83-129.22)^{2} \\
& +(252.83-120.7)^{2}+(252.83-139.82)^{2} \\
& +(198.43-171.46)^{2}+(198.43-154.11)^{2} \\
& +(198.43-129.22)^{2}+(198.43-120.7)^{2} \\
& +(198.43-139.82)^{2}+(171.46-154.11)^{2} \\
& +(171.46-129.22)^{2}+(171.46-120.7)^{2} \\
& +(171.46-139.82)^{2}+(154.11-129.22)^{2} \\
& +(154.11-120.7)^{2}+(154.11-139.82)^{2} \\
& +(129.22-120.7)^{2}+(129.22-139.82)^{2} \\
& +(120.7-139.82)^{2}=89949.91 \mathrm{~dB}^{2} .
\end{aligned}
$$

Contribution ratio in $\%$ of $M_{W}$

$$
\begin{aligned}
=89949.91 /( & 4489.1+1270.8+1406.7+4677.6 \\
& +21419.2+4335+89949.9+8539.7) \\
\times 100 \%= & 66.10 \% .
\end{aligned}
$$

Table 2 shows the Pareto-ANOVA analysis details. It is found that the contribution ratio of $M_{w}$ and $Z_{w}$ is significant compared to those of other parameters, showing higher sensitivity of these parameters. Also, this analysis gives the optimum combination of parameters to reduce the sensitivity. For each parameter, the level which gives the highest SNR is the optimum level.

From Table 2, it can be seen that the optimum levels of parameters $X_{\dot{u}}, Z_{\dot{w}}, Z_{\dot{q}}, M_{\dot{q}}, Z_{w}, Z_{q}, M_{w}$, and $M_{q}$ are 2, 2, 4, 6, $7,1,1$, and 5 , respectively. The corresponding physical values are $25 \%, 25 \%, 55 \%, 85 \%, 100 \%, 10 \%, 10 \%$, and $70 \%$ of their original values. 


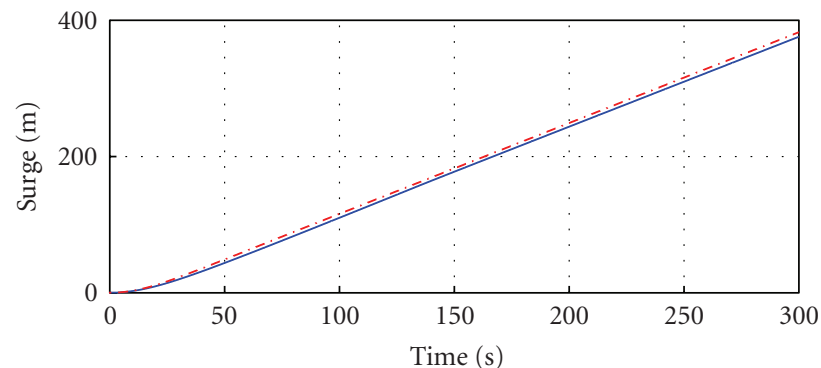

(a)

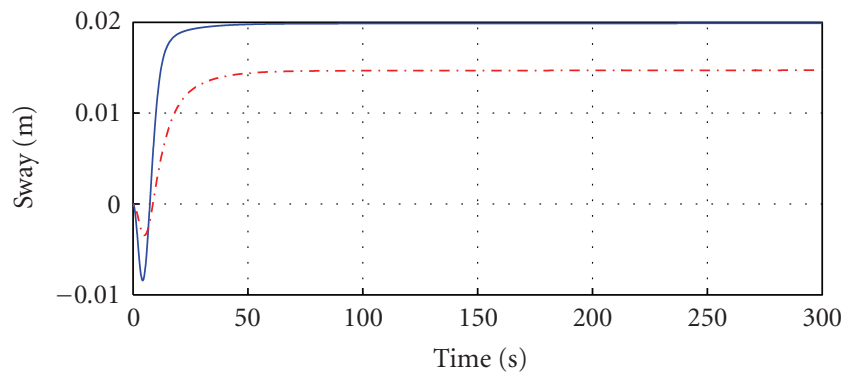

(c)

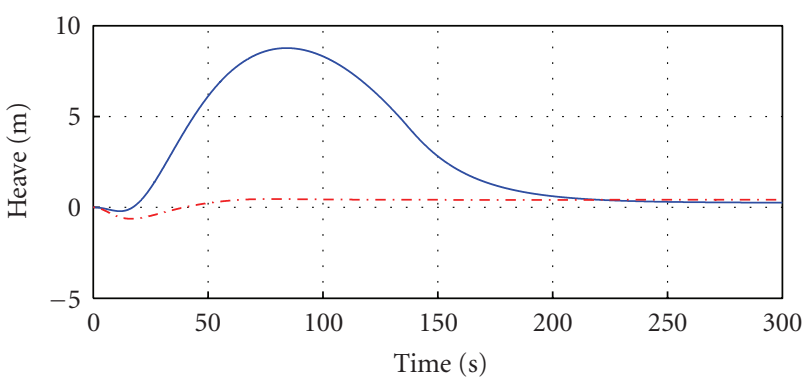

- Original
-. Optimized

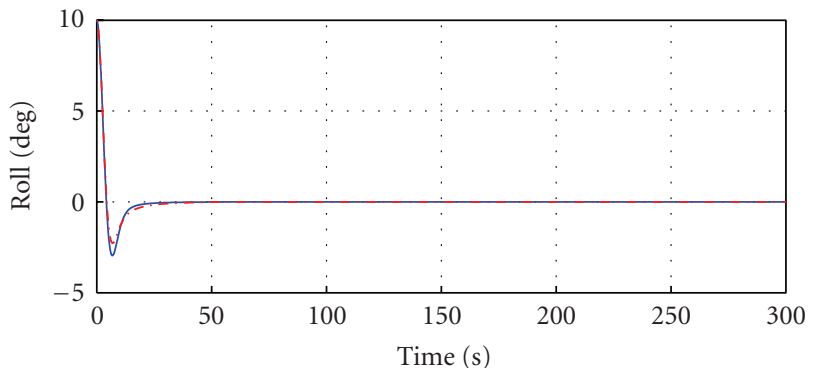

(b)

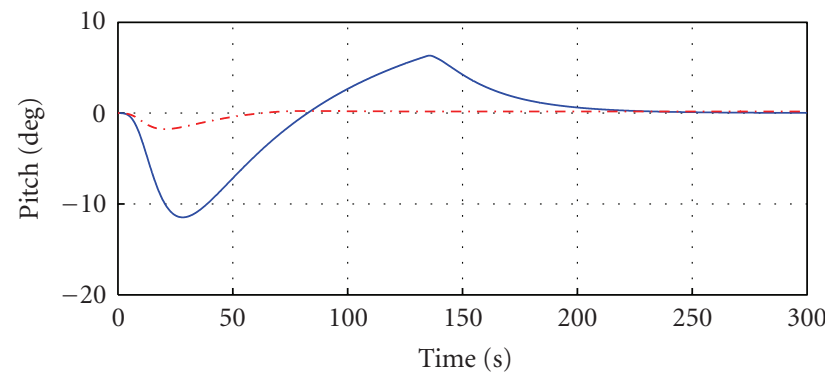

(d)

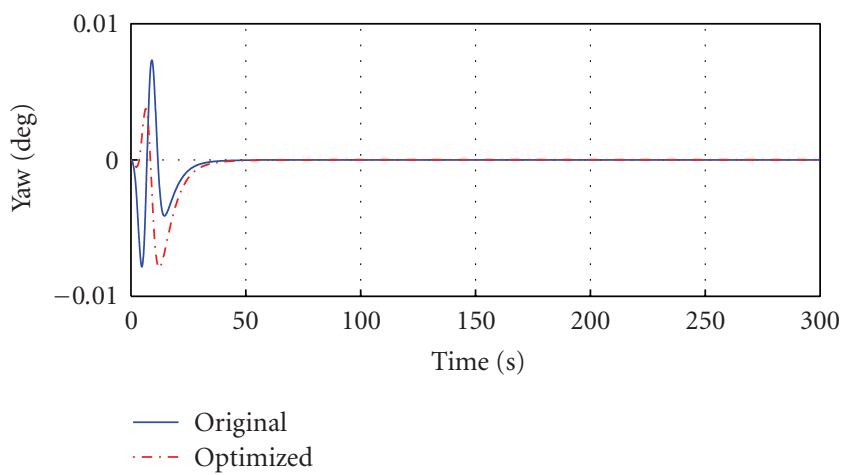

(f)

FIgURE 6: Comparative results of positions and orientations for closed loop control.

TABLe 2: Pareto-ANOVA analysis.

\begin{tabular}{lccccccccc}
\hline \multirow{2}{*}{ Parameters } & \multicolumn{9}{c}{ Sum of S/N ratio, $S_{i j}$} \\
& level 1 & level 2 & level 3 & level 4 & level 5 & level 6 & level 7 & Sum of squares of differences & \multirow{2}{*}{ Contribution ratio in \% } \\
\hline$X_{\dot{u}}$ & 167.70 & $\mathbf{1 8 6 . 4 0}$ & 168.68 & 157.08 & 154.62 & 163.92 & 168.18 & 4489.094 & 3.30 \\
$Z_{\dot{w}}$ & 166.50 & $\mathbf{1 7 3 . 4 5}$ & 155.62 & 165.76 & 169.65 & 167.06 & 168.55 & 1270.777 & 0.93 \\
$Z_{\dot{q}}$ & 167.05 & 169.15 & 155.09 & $\mathbf{1 7 3 . 8 4}$ & 164.79 & 168.10 & 168.55 & 1406.652 & 1.03 \\
$M_{\dot{q}}$ & 168.32 & 154.93 & 165.08 & 168.29 & 170.19 & $\mathbf{1 8 5 . 5 3}$ & 154.24 & 4677.602 & 3.44 \\
$Z_{w}$ & 128.71 & 140.81 & 177.19 & 171.52 & 182.14 & 178.84 & $\mathbf{1 8 7 . 3 7}$ & 21419.23 & $\mathbf{1 5 . 7 4}$ \\
$Z_{q}$ & $\mathbf{1 8 6 . 7 6}$ & 156.14 & 156.99 & 163.89 & 166.88 & 167.84 & 168.07 & 4335.005 & 3.19 \\
$M_{w}$ & $\mathbf{2 5 2 . 8 3}$ & 198.43 & 171.46 & 154.11 & 129.22 & 120.70 & 139.82 & 89949.91 & $\mathbf{6 6 . 1 0}$ \\
$M_{q}$ & 138.93 & 167.11 & 167.65 & 168.93 & $\mathbf{1 8 7 . 5 8}$ & 169.23 & 167.16 & 8539.692 & 6.28 \\
\hline
\end{tabular}

TABle 3: Comparative results.

\begin{tabular}{|c|c|c|c|c|c|c|c|c|c|c|c|}
\hline \multirow{2}{*}{ Condition } & \multirow{2}{*}{$\begin{array}{c}X_{\dot{u}} \\
(\%) \\
\end{array}$} & \multirow{2}{*}{$\begin{array}{c}Z_{\dot{w}} \\
(\%)\end{array}$} & \multirow{2}{*}{$\begin{array}{c}Z_{\dot{q}} \\
(\%)\end{array}$} & \multirow{2}{*}{$\begin{array}{l}M_{\dot{q}} \\
(\%)\end{array}$} & \multirow{2}{*}{$\begin{array}{c}Z_{w} \\
(\%)\end{array}$} & \multirow{2}{*}{$\begin{array}{c}Z_{q} \\
(\%)\end{array}$} & \multirow{2}{*}{$\begin{array}{l}M_{w} \\
(\%)\end{array}$} & \multirow{2}{*}{$\begin{array}{l}M_{q} \\
(\%)\end{array}$} & \multicolumn{3}{|c|}{ Pitch angle (deg) for $U=$} \\
\hline & & & & & & & & & $1.13(\mathrm{~m} / \mathrm{s})$ & $1.59(\mathrm{~m} / \mathrm{s})$ & $1.98(\mathrm{~m} / \mathrm{s})$ \\
\hline Original values & 100 & 100 & 100 & 100 & 100 & 100 & 100 & 100 & -25.13 & -25.46 & -25.62 \\
\hline Optimized values & 25 & 25 & 55 & 85 & 100 & 10 & 10 & 70 & -2.86 & -2.92 & -2.95 \\
\hline
\end{tabular}


In order to show the effectiveness of this analysis in identifying optimum parameters to reduce the pitch angle variations of the AUV, simulations were carried out using the optimal values of the hydrodynamic parameters. The simulation results are summarized in Table 3 and results are shown in Figures 5 and 6 . It was observed that the pitch angle error has significantly been reduced by the use of optimized values. As a result, the errors in heave, surge, sway, and yaw were also reduced. Similarly, the closed loop control results show improved transient and steady state response (Figure 6). Further fine tuning of the parameter values may still reduce the errors to almost zero.

The significance of the analysis is in the redesign of the AUV based on the sensitivity analysis. Once the most sensitive parameters were identified, designer can focus his attention on redesigning the physical features of the AUV to achieve the desired values for these parameters. For example, additional control planes in the aft or increased control plane areas will reduce the hydrodynamic moment $\left(M_{w}\right)$ in the heave motion.

\section{Conclusion}

A systematic study on the sensitivity of various hydrodynamic parameters on the performance of an underwater robot was presented. Using statistical design techniques, critical parameters affecting the diving plane motion of the robot were identified and optimal combination of parameters to reduce the pitch angle error was determined. Simulation results have shown the effectiveness of the analysis in improving the dynamic performance. Further studies may include the robot motion in a three-dimensional plane and the sensitivity of environmental disturbance parameters as noise variables. Experimental validation of above findings will be taken up as soon as the prototype AUV is ready for trials.

\section{Acknowledgments}

The authors wish to thank the editor and the anonymous referees for their valuable comments and suggestions that improved the paper.

\section{References}

[1] D. Perrault, N. Bose, S. O'Young, and C. D. Williams, "Sensitivity of AUV added mass coefficients to variations in hull and control plane geometry," Ocean Engineering, vol. 30, no. 5, pp. 645-671, 2003.

[2] D. Perrault, N. Bose, S. O'Young, and C. D. Williams, "Sensitivity of AUV response to variations in hydrodynamic parameters," Ocean Engineering, vol. 30, no. 6, pp. 779-811, 2003.

[3] C. V. Alt, "Autonomous underwater vehicles," in Proceedings of the Autonomous Underwater Lagrangian Platforms and Sensors Workshop, pp. 1-5, Sea Lodge, La Jolla, Calif, USA, March 2003.

[4] C. Aage and L. W. Smitt, "Hydrodynamic maneuverability data of a flatfish type AUR," in Proceedings of the IEEE
Oceans Engineering for Today's Technology and Tomorrow's Preservation, vol. 3, pp. 425-430, Brest, France, September 1994.

[5] T. I. Fossen, Guidance and Control of Ocean Vehicles, John Wiley \& Sons, England, UK, 1994.

[6] M. Santhakumar, T. Asokan, and T. R. Sreeram, "A 6 sigma framework for the design of flatfish type autonomous underwater vehicle (AUV)," in Proceedings of the SAE World Congress and Exhibition, SAE Paper Series, Detroit, Mich, USA, April 2009.

[7] S. H. Park, Robust Design Analysis for Quality Engineering, Chapman \& Hall, London, UK, 1996.

[8] P. R. Rosenbaum, "Sensitivity analysis in observational studies," Encyclopedia of Statistics in Behavioral Science, vol. 4, pp. 1809-1814, 2005.

[9] H. C. Frey and S. R. Patil, "Identification and review of sensitivity analysis methods," Risk Analysis, vol. 22, no. 3, pp. 553-578, 2002.

[10] M. Phadke, Quality Engineering Using Robust Design, PrenticeHall, Englewood Cliffs, NJ, USA, 1989.

[11] D. M. Byrne and S. Taguchi, "The taguchi approach to parameter design," in Proceedings of the 40th Quality Congress Transactions, vol. 40, pp. 168-177, Anaheim, Calif, USA, May 1986.

[12] R. N. Kackar, "Off-line quality control, parameter design, and the Taguchi approach," Journal of Quality Technology, vol. 4, pp. 17-24, 1985.

[13] G. Box, "Signal-to-noise ratios, performance criteria, and transformations," Technometrics, vol. 30, no. 1, pp. 1-17, 1988.

[14] P. J. Ross, Taguchi Techniques for Quality Engineering, McGraw-Hill, New York, NY, USA, 1988.

[15] K. Al-Widyan and J. Angeles, "A model-based formulation of robust design," ASME Journal of Mechanical Design, vol. 127, no. 3, pp. 388-396, 2005.

[16] Y. Wu and A. Wu, Taguchi Methods for Robust Design, ASME, New York, NY, USA, 2000. 

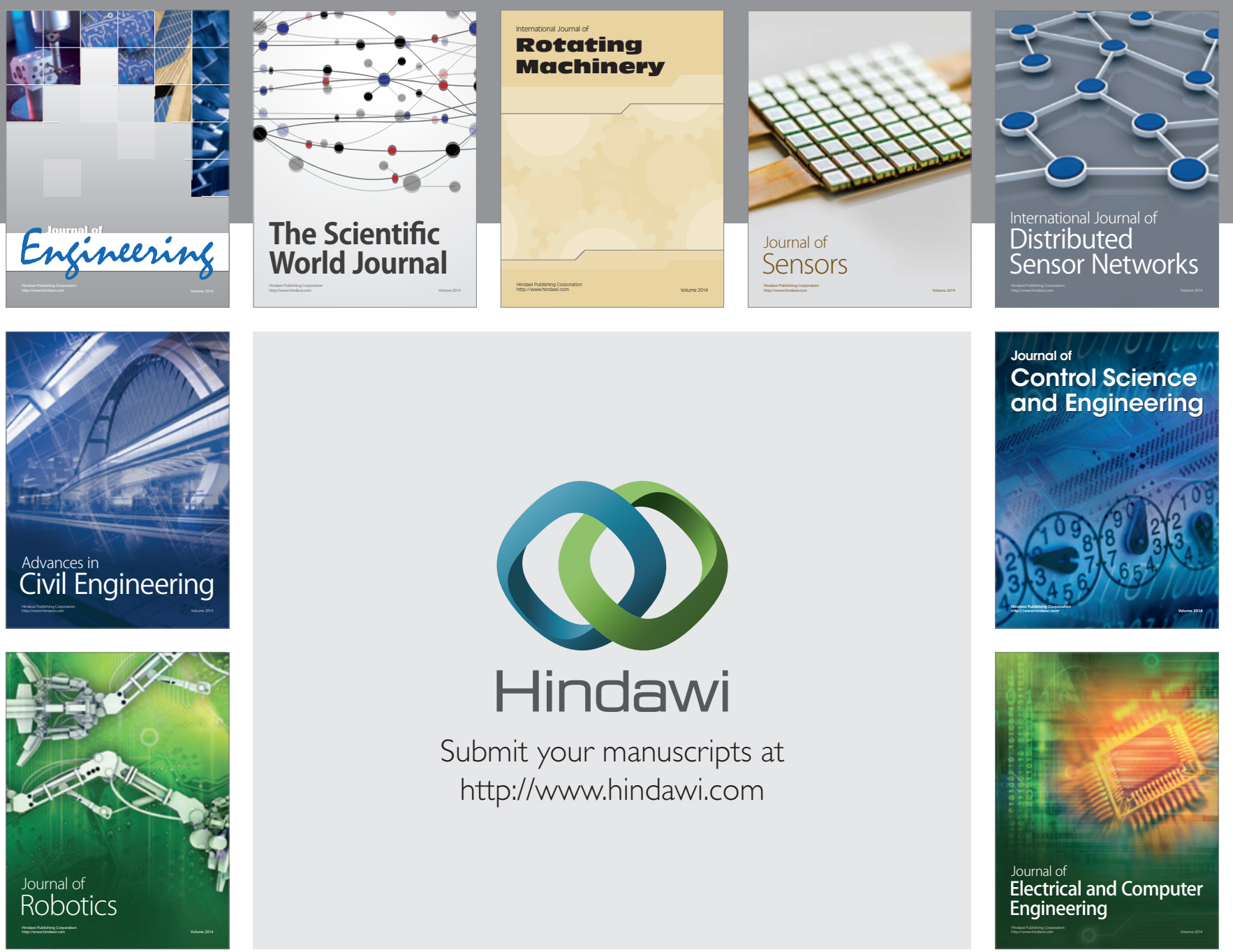

Submit your manuscripts at

http://www.hindawi.com
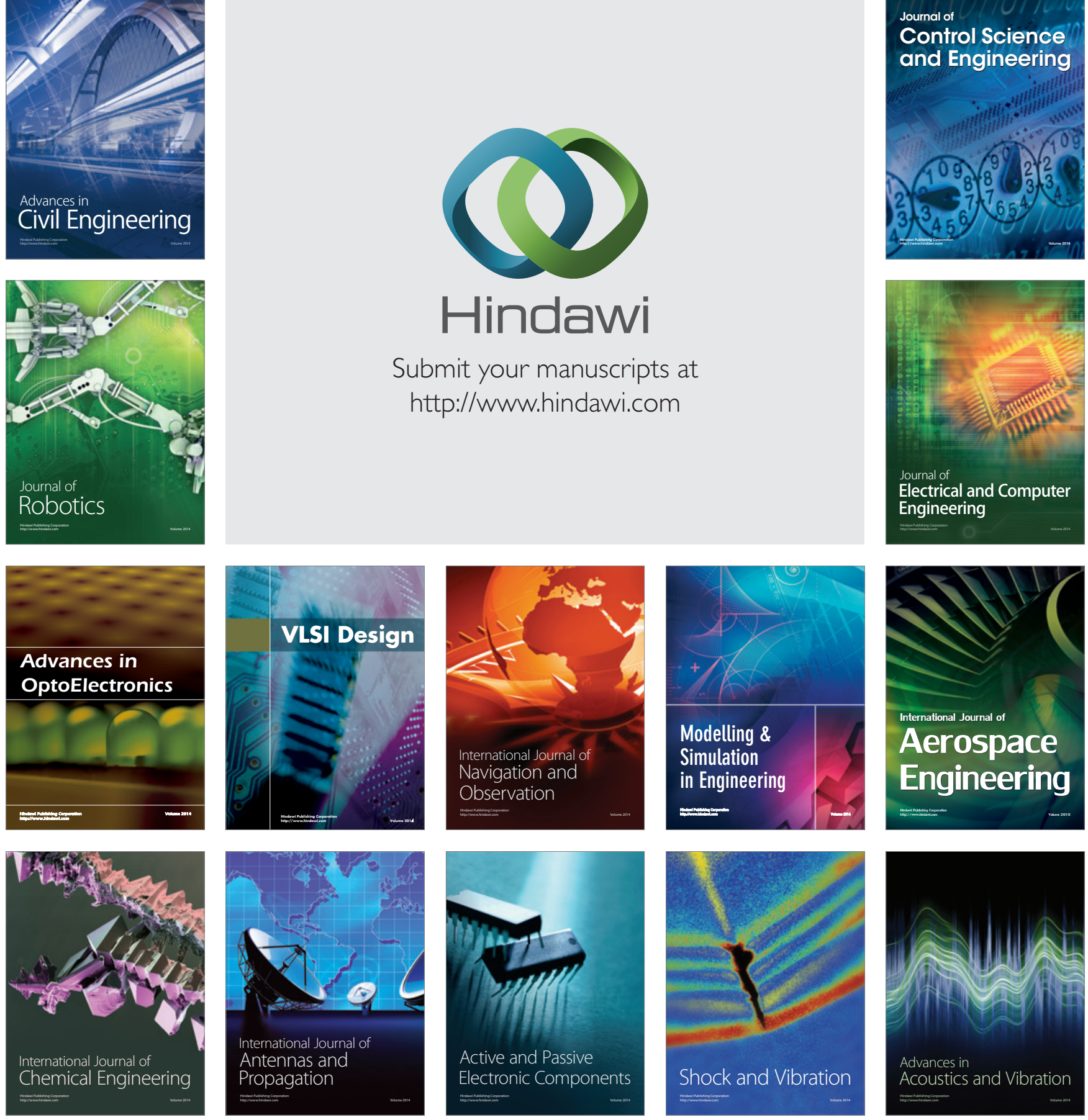